\title{
Comparison of lossy filters and predistorted filters using novel software
}

\author{
Alberto Padilla ${ }^{1}$, Jordi Mateu ${ }^{1,2}$, Carlos Collado ${ }^{1}$, Christoph Ernst ${ }^{3}$, Juan M. Rius ${ }^{1}$, Jose M. Tamayo ${ }^{1}, \quad$ Juan \\ M. O'Callaghan ${ }^{1}$ \\ ${ }^{1}$ Universitat Politècnica de Catalunya (UPC), Campus Nord, Barcelona, Spain \\ ${ }^{2}$ Centre Tecnològic de Telecomunicacions de Catalunya (CTTC), PMT, Castelldefels, Spain \\ ${ }^{3}$ European Space Agency (ESA), ESTEC, Noordwijk ZH, The Netherlands
}

\begin{abstract}
This paper compares several synthesis alternatives for a $4 \mathrm{GHz}$ filter with $36 \mathrm{MHz}$ bandwidth. The alternatives considered are: classical synthesis, various forms of predistortion and lossy filter synthesis. Electrical performance of the various alternatives is discussed in detail and a brief description of the topologies is also given. The synthesis of the several alternatives presented in this paper has been performed with a novel software that generates the desired transfer response, obtains the filter transverse coupling matrix and transforms it into other topologies of technological interest. The paper includes a brief description of the software features.
\end{abstract}

Index Terms - Lossy filter, insertion loss, synthesis, matrix rotation, channelizing filters.

\section{INTRODUCTION}

Microwave passive filters play a very important role in almost any communication system. These filters are implemented in many different technologies usually depending on their electric and mechanical requirements. Although their design is strongly dependent on the technology used for their implementation, the synthesis procedure is usually common for all. This classical synthesis procedure consists on obtaining a purely reactive (no dissipation effect) network that defines the resonant frequency of the resonators forming the filter and the way they are coupled [1]. However, in practice, some dissipation always exist in the final filter implementation which can be evaluated afterwards by introducing the material losses (finite $\mathrm{Q}$ of the resonators) into the synthesized lossless network. Among other effects, this approach leads to filters with minimum insertion loss in the passband at one frequency at the expense of additional passband rounding towards the band-edges, being the use of high $\mathrm{Q}$ resonators the only way to achieve filters with flat pass-band response by means of classical synthesis techniques [1]. This may result in heavy and large filters which might be impractical in space systems, or in wireless and handset components with strong demand for low-cost and small size.

This can be overcome by the use of non standard filter synthesis techniques such as lossy filter synthesis [2]-[5] and/or (adaptive) pre-distortion techniques [1],[6]. These techniques allow obtaining good insertion loss flatness with low Qs at the expense of either return loss (pre-distortion) or absolute insertion loss (lossy filters). The latter may be useful in receiver architectures where filter absolute insertion loss is not critical, such as those where the filtering and signal channelization are performed after the low noise amplifier, as it occurs in input multiplexers (IMUX) for satellite transponders [1].

The critical parameters in IMUX channelizing filters are the insertion loss flatness, the selectivity of the transfer function and the out of band return loss at the input, which has to be low (reflective). Additionally, good in-band return loss at the input is necessary for manifold-type IMUX and may somewhat relax the rejection specifications of some of the filters in IMUX with circulator chain [1],[7].

This paper will describe a preliminary study of the feasibility of using lossy filter synthesis in achieving several of the key features needed for IMUX channelizing filters. We will compare several synthesis alternatives, including classical synthesis, pre-distortion and lossy filter synthesis; and show their relative merits in terms of return loss, absolute insertion loss and insertion loss flatness. The main features of the software used for this study will also be discussed.

\section{SPECIFICATIONS AND DESIGN ALTERNATIVES}

This section outlines the several design alternatives used for the comparison performed in this work. All the filter responses evaluated here have a sixth order Chebyshev frequency response centered at $4 \mathrm{GHz}$ with $36 \mathrm{MHz}$ bandwidth and a single pair of symmetric transmission zeros at $3.875 \mathrm{GHz}$ and $4.129 \mathrm{GHz}$, respectively, which correspond to a normalized frequency of \pm 1.7 (see Table I). These relaxed specifications are set to produce a simple breadboard that demonstrates the basic desired features of IMUX channelizer filters before facing the difficulties that a more realistic set of specifications would bring (among other issues, group delay specifications are omitted).

\begin{tabular}{lcl}
\hline \hline Parameter & Value & UNIT \\
\hline Center frequency $\left(\mathrm{f}_{0}\right)$ & 4000 & $\mathrm{MHz}$ \\
\hline Channel Bandwidth & 36 & $\mathrm{MHz}$ \\
\hline Insertion loss over bandwidth & $<6.6$ & $\mathrm{~dB}$ \\
\hline Insertion loss at center frequency & $<6$ & $\mathrm{~dB}$ \\
\hline Input and Output Match & 18 & $\mathrm{~dB}$ \\
\hline \multicolumn{2}{c}{ In-band Insertion loss variation } \\
\hline F0+/-13 MHz & 0.2 & $\mathrm{dBpp}$ \\
\hline $\mathrm{F} 0+/-16 \mathrm{MHz}$ & 0.3 & $\mathrm{dBpp}$ \\
\hline $\mathrm{F} 0+/-18 \mathrm{MHz}$ & 0.6 & $\mathrm{dBpp}$ \\
\hline \hline
\end{tabular}

Table I: Specification of the Channelizing filter used in this work. 
The paragraphs below describe the design alternatives to be compared and introduce the acronyms that will be used to refer to them.

CONV: Filter synthesized using conventional (lossless) synthesis techniques. This filter is the baseline to design the other filters that use either pre-distortion or lossy filter synthesis, and is also used as a baseline for comparing frequency responses of the various alternatives. The required resonator $\mathrm{Q}$ for this design was found by evaluating its frequency response for various values of $Q$ and finding the lowest one compatible with the requirements on insertion loss flatness [1].

FPD1: Filter synthesized using full pre-distortion techniques assuming resonator Qs of 3000 (technologically available in combline). Full predistortion techniques recover the in band flatness of the filter transmission response by reflecting energy in the passband. This is achieved in the synthesis by moving the poles of the characteristic polynomials from their position in the lossless case by an amount $\sigma=1 /\left(F B W \cdot Q_{0}\right)$ where FBW is the fractional bandwith and $Q o$ the resonator Q [1]. The starting point for the pre-distortion procedure is the CONV design above.

FPD2: Filter synthesized using full pre-distortion techniques assuming resonator Qs of 1600 (the minimum required to satisfy the IL specs of table I). The starting point for the pre-distortion procedure is the CONV design above [1].

PPD: Filter synthesized using partial pre-distortion and resonator $\mathrm{Q}_{0}=3000$ (technologically available). Pre-distortion is used to emulate a response with an effective resonator $Q$ $\left(Q_{\text {eff }}\right)$ of 6000 , necessary to fulfill the insertion loss flatness requirements. This can be achieved by moving the poles of the transfer function by $\sigma=1 / F B W\left(\frac{1}{Q_{0}}-\frac{1}{Q_{e f f}}\right)$ [6].The starting point for the pre-distortion procedure is the CONV design above [1].

APD: Filter synthesized using adaptive pre-distortion and resonator $\mathrm{Q}=3000$ (technologically available). In this synthesis the poles of the transfer function are moved following a sinusoidal shape [6]. The starting point for the pre-distortion procedure is the CONV design.

LF: Filter synthesized using lossy filter synthesis techniques for an insertion loss of $3 \mathrm{~dB}$ and resulting in a passband return loss of $18 \mathrm{~dB}$. This approach flattens the pass band of the transmission response by absorption of energy instead of reflection as a predistortion case. This synthesis was presented in [3] and fully detailed in [11]. The resulting passband flatness is that of a lossless filter, which is much better than that set in Table I. The starting point for this design is the CONV design.

LF_QR: Filter synthesized using lossy filter synthesis techniques (as in LF above), but whose resonator Qs have been reduced to match the insertion loss flatness requirements of Table I.

LF_OPT: Filter designed according to LF whose design has undergone computer optimization to reduce the $\mathrm{Q}$ requirements and improve passband return loss to $21 \mathrm{~dB}$.

CONV_1: Filter synthesized using conventional (lossless) synthesis techniques and $21 \mathrm{~dB}$ of passband return loss (unlike CONV above). Once synthesized, the frequency response is evaluated for a $\mathrm{Q}$ of 3000 as the one used in the optimized lossy filter (LF_OPT). The resulting synthesis does not fulfill the specifications on passband flatness. This design is used as a baseline for comparison against LF and LF_OPT.

\section{SOFTWARE TOOL}

The various filter design alternatives outlined in Section II have been synthesized using a software tool developed for the study of a new class of receiver filters [9]-[11]. The software is capable of synthesizing a transversal matrix corresponding to a network having either Butterworth or generalized Chebyshev responses, with the optional inclusion of transmission and group delay equalization zeroes. This is done using either conventional (lossless), pre-distortion or lossy synthesis, in all the variants listed in Sect. II. The resulting transversal coupling matrix can then be transformed into more appropriate topologies for the final filter implementation. For the most common configurations, such as the folded topology, automatic circuit transformation is already implemented in the software. Additional circuit transformations can be defined by the user by means of trigonometric and/or hyperbolic rotation and node scaling.

For the particular case of lossy filters specific topologies with a uniform distribution of the losses can be also performed with the software.

Additionally the software also allows the optimization of the coupling matrix value for a better fulfillment of the desired specifications.

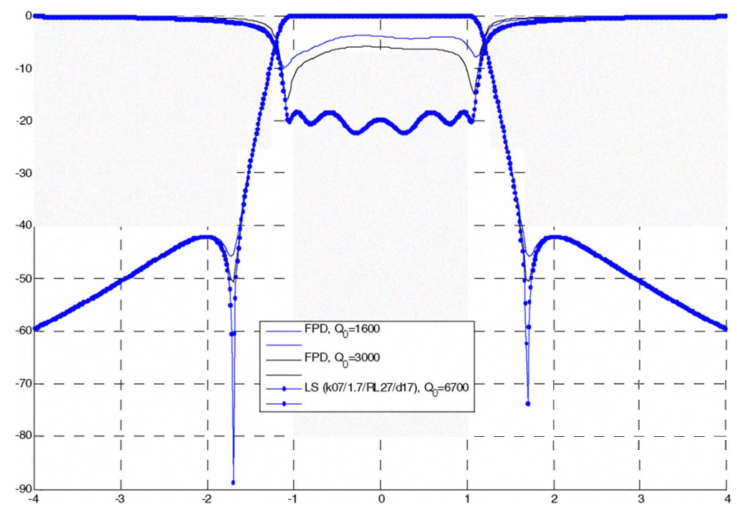

Fig. 1- Frequency responses (transmission and reflection) of design alternatives LF (blue traces with circles), FPD1 (black traces) and FPD2 (plain and dashed blue traces) Note that transmission responses are normalized to $0 \mathrm{~dB}$ in the passband, See Table II for passband insertion loss (IL) values. 


\section{COMPARISON OF SYNTHESIS METHODS}

This section compares the frequency responses of the different alternatives outlined in Sect. II whose main features are listed in Table II.

\begin{tabular}{|c|c|c|c|c|c|c|c|c|}
\hline Filter & $\begin{array}{c}\text { IL } \\
\text { (dB) }\end{array}$ & $\begin{array}{l}\text { RTL } \\
\text { (dB) }\end{array}$ & $\begin{array}{c}\text { Flatness } \\
\text { (dB) }\end{array}$ & \begin{tabular}{|c|} 
Sel \\
\pm 23 \\
MHz \\
\end{tabular} & \begin{tabular}{|c|} 
Sel \\
\pm 30-500 \\
MHz \\
\end{tabular} & \begin{tabular}{|c|} 
GD \\
$10 \mathrm{MHz}$ \\
(ns) \\
\end{tabular} & $\begin{array}{c}\text { GD } \\
18 M H z \\
\text { (ns) } \\
\end{array}$ & $\mathbf{Q}$ \\
\hline CONV & 0.41 & 27 & 0.6 & 10.6 & \begin{tabular}{|l|}
42 \\
\end{tabular} & 4.2 & 31 & 6000 \\
\hline FPD1 & 2.7 & 6.2 & 0.01 & 10.5 & 42 & 4.1 & 30.4 & 3000 \\
\hline FPD2 & 6.3 & 4 & 0.01 & 10.3 & 41.9 & 4 & 30 & $\begin{array}{c}1600 \\
\text { Min. Q } \\
\mathrm{IL}=6 \mathrm{~dB} \\
\end{array}$ \\
\hline PPD & 1.7 & 9.4 & 0.6 & 10.3 & 41.7 & 4.1 & 29.9 & $\begin{array}{c}3000 \\
\left(Q_{\text {eff }}=6000\right)\end{array}$ \\
\hline APD & 2.1 & 7.8 & 0.29 & 10.5 & 42 & 4.2 & 30.3 & 3000 \\
\hline $\mathbf{L F}$ & 3 & 18 & 0.01 & 10.6 & 42.1 & 4.2 & 30.7 & $\begin{array}{c}6700 \\
800 \\
\text { (In/output) }\end{array}$ \\
\hline LF_QR & 2.67 & 18 & 0.46 & 10.3 & 42 & 4.2 & 29.12 & $\begin{array}{c}3500 \\
2800 \\
\text { (In/output) } \\
\left(\mathrm{Q}_{\mathrm{eff}}=8000\right)\end{array}$ \\
\hline LF_OPT & 5.5 & 21 & 0.2 & 10.5 & 40 & 6 & 41 & 3000 \\
\hline CONV1 & 1.2 & 21 & 1.6 & 15.8 & 46 & 4.2 & 31 & 3000 \\
\hline
\end{tabular}

Table II: Comparison of the frequency response of the filters.

Figure 1 shows the frequency response of the design obtained using lossy filter synthesis (LF) and its comparison with the designs using full pre-distortion (FPD1, FPD2). Among all the alternatives explored, these are the designs with better insertion loss flatness i.e., their flatness is that of an ideal lossless response (and better than the specifications). None of the two pre-distortion alternatives fulfill the return loss requirement. However, these are the alternatives with the smaller required Q (Table II). The lossy filter synthesis, on the other hand, produces much better return loss than predistortion, at the expense of requiring higher Qs.

Fig. 2 shows variants of the pre-distortion (PPD and APD) and lossy filter synthesis (LF_QR) producing transfer responses whose insertion loss flatness is closer to the specifications. According to Table II, this improves the matching of the pre-distortion alternatives (PPD and APD) with respect to those in Fig. 1 (FPD1, FPD2) whereas, the lossy filter solution (LF_QR) shows a significant reduction in required $\mathrm{Q}$ with respect to that in Fig. 1 (LF).

At this stage we focus our attention to the lossy filter alternatives as the only ones (among those listed in Sect. II) capable of providing the required return loss and, at the same time, are able to produce the required selectivity and insertion losss flatness with the resonator $Q$ s available $(Q=3000)$. To further improve on the designs LF and LF_QR, we produced design LF_OPT through computer optimization to improve on the return loss and $\mathrm{Q}$ requirements. We also synthesized the CONV_1 design to have an updated comparison baseline with a conventional synthesis having equal return loss to the one achieved in LF_OPT. Figure 3 and Table II show the comparison between LF, LF_OPT and CONV_1. Note that the latter does not fulfill the requirements in insertion loss flatness, whereas LF_OPT is compliant and -at the same timehas better return loss and $Q$ requirements that the initial lossy filter synthesis (LF).

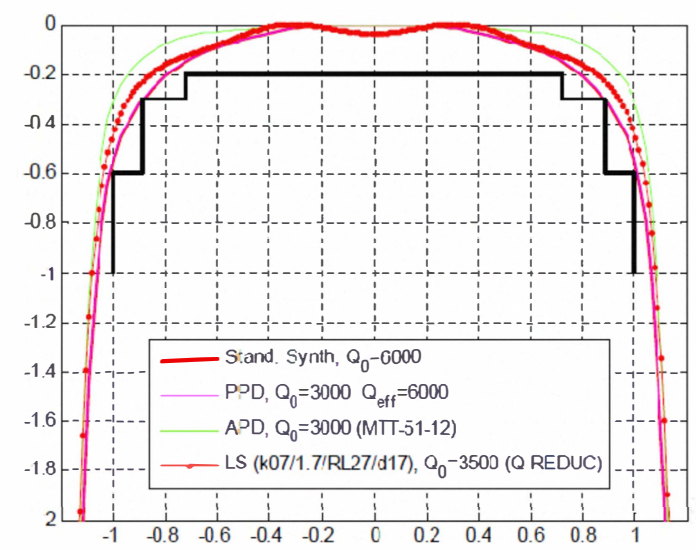

Fig. 2- Zoom-in (0-2dB) of the transfer function of the following design alternatives: CONV (red trace), PPD (pink trace), APD (green trace) and LF_QR (red trace with circles). Note that CONV and PPD traces are overlapped. Transmission responses are normalized with respect to the minimum insertion loss. The black lines indicate the template in the transmission response.

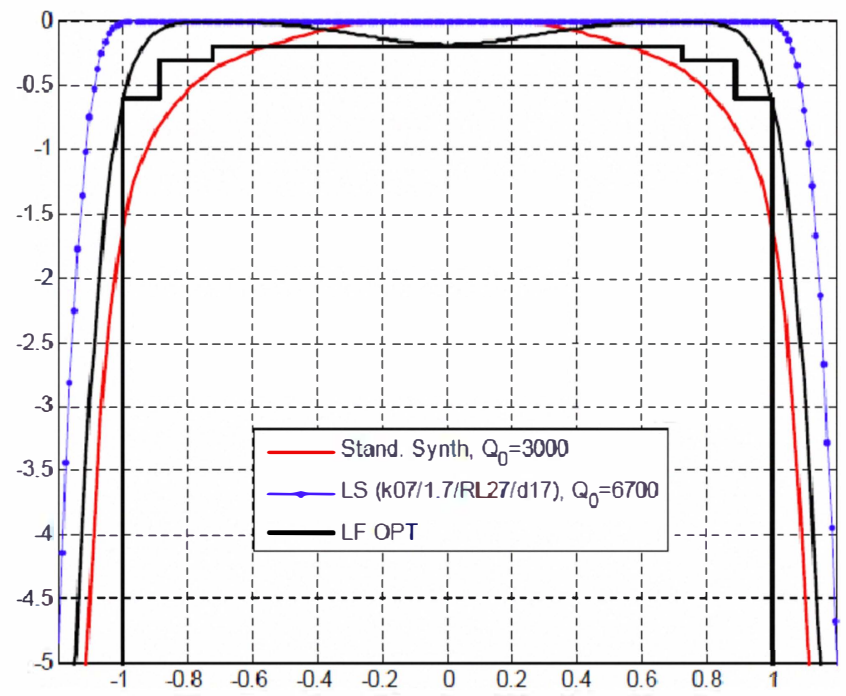

Fig. 3-Comparison between the frequency response of the envisaged synthesis alternative LF_OPT (black trace), the lossless design with $21 \mathrm{db}$ return loss (CONV1, red traces) and that of the original design using lossy filter synthesis LF (blue traces)). The straight black lines show the specs template for the transmission response.

Table III summarizes the advantages and disadvantages of pre-distortion vs. lossy filters according to the examples described in this paper. The table also shows the topologies used for the lossy filter solutions and for the ones using pre- 
distortion. For the lossy filter solutions, the Qs required in the input and output resonators (pink background in Table III) are different than those of the inner resonators (orange background in Table III) for designs LF and LF_QR (see Table II), whereas LF_OPT has uniform $Q$ requirements $(\mathrm{Q}=3000)$. All pre-distortion designs have uniform requirements in $\mathrm{Q}$ in their resonators (Table II). Another important difference in the topologies described in Table III is the requirement for resistive couplings for the lossy filter designs [2][3].

\begin{tabular}{|c|c|c|c|}
\hline SYNTHESIS TYPE & \multicolumn{2}{|c|}{ ADVANTAGES } & "DISAVANTAGES \\
\hline $\begin{array}{c}\text { (Partial / Adaptive) } \\
\text { Predistortion } \\
\text { Techniques }\end{array}$ & \multicolumn{2}{|c|}{$\begin{array}{l}\text { - Uniform Q } \\
\text { - Lower Q } \\
\text { - No resistive } \\
\text { couplings } \\
\text { - Folded topology } \\
\end{array}$} & $\begin{array}{l}\text { - Poor RTL } \\
\text { - TZ less distinct }\end{array}$ \\
\hline $\begin{array}{l}\text { Lossy synthesis } \\
\text { techniques }\end{array}$ & \multicolumn{2}{|c|}{$\begin{array}{l}\text { - Higher Selectivity } \\
\text { - Better RTL } \\
\text { - Improved GD (No } \\
\text { self-equalization) } \\
\text { - Folded topology }\end{array}$} & $\begin{array}{l}\text { - Almost Uniform Q } \\
\text { - Requires resistive } \\
\text { couplings }\end{array}$ \\
\hline \multicolumn{4}{|c|}{$\begin{array}{ll}\text { TOPOLOGIES } \\
\end{array}$} \\
\hline \multicolumn{2}{|c|}{$\begin{array}{c}\text { (Partial / Adaptive) } \\
\text { Predistortion Techniques }\end{array}$} & \multicolumn{2}{|c|}{ Lossy synthesis techniques } \\
\hline$\gamma$ & & & \\
\hline
\end{tabular}

Table III: Comparison between predistortion and lossy filter responses and topologies.

\section{CONCLUSION}

Lossy filter synthesis combined with computer optimization has been compared to other synthesis alternatives and has been found to yield frequency responses with improved performance in several parameters which are relevant in satellite IMUX. In particular, among the alternatives explored, LF_OPT is the only one achieving all the requirements in the specifications (including return loss) with the $\mathrm{Q}$ available in the technology envisaged (combline). All the design alternatives presented in this work have been synthesized using a software tool developed in house. The resulting topologies presented in Table III have also been obtained by the use of this software. Our next step envisaged is to design a demonstration prototype to address the technological hurdles for the implementation of this type of synthesis.

\section{ACKNOWLEDGMENTS}

This work was supported by ESA under the contract AO 1$5477 / 07 / \mathrm{NL} / \mathrm{GLC}$, by Spanish Ministry (SM) of Science and Innovation through grants TEC-2006-13248-C04-02/TCM and MAT2008-06761-C03-02; and Spanish Ministry of Education through a PhD fellowship for A. Padilla (AP200802235).

\section{REFERENCES}

[1] R. Cameron, C. M. Kudsia, R. R. Mansour, "Microwave filters for communication systems" John Wiley, 2007.

[2] M. Yu, V. Miraftab, "Shrinking Microwave Filters" IEEE Microwave Magazine, October 2005, pp. 40-54.

[3] I. Hunter, A. Guyette, R. D. Pollard, "Passive Microwave Receive Filter Networks Using Low-Q Resonators", IEEE Microwave Magazine, Sept. 2005

[4] Miraftab, V.; Ming Yu, "Generalized Lossy Microwave Filter Coupling Matrix Synthesis and Design Using Mixed Technologies," Microwave Theory and Techniques, IEEE Transactions on, vol.56, no. 12, pp.3016-3027, Dec. 2008.

[5] Miraftab, V.; Ming Yu, "Advanced Coupling Matrix and Admittance Function Synthesis Techniques for Dissipative Microwave Filters," Microwave Theory and Techniques, IEEE Transactions on, vol.57, no.10, pp.2429-2438, Oct. 2009

[6] M. Yu, W-C Tang, A. Malarky, V. Dokas, R. Cameron, Y. Wang, "Predistortion Technique for Cross-Coupled Filters and its application to satellite communication systems", IEEE Trans. On Microwave Theory and Techniques, vol. 51, No.12, Dec. 2003.

[7] T. Kässer. Final Report. "Minimux". Miniaturized Ku-Band Input Multiplexer. ESA-Program AO 4396, Multimedia Satellite Equipment

[8] F.-J. Görtz. Executive Summary. "New Generation Input Demultiplexer NGIMUX". ESA Program AO 4184 Multimedia Satellite Equipment.

[9] www.tsc.upc.edu/lossyfilters

[10] European Space Agency. Statement of Work Design and Synthesis of a New Class of Receiver Filters (AO/15477/07/NL/GLC).

[11] J. Mateu, C. Collado, J.M. O’Callaghan. Lossy filter synthesis and study of topology solution space. ESA working paper 2366. TEC-ETM/2009. 